\title{
Genomic Profiling in Luminal Breast Cancer
}

\author{
Oleg Gluz ${ }^{\mathrm{a}}$ Daniel Hofmann ${ }^{\mathrm{a}} \quad$ Rachel Würstlein ${ }^{\mathrm{b}} \quad$ Cornelia Liedtke ${ }^{\mathrm{c}}$ \\ Ulrike Nitz ${ }^{\mathrm{a}}$ Nadia Harbeck ${ }^{\mathrm{b}}$ \\ aEvangelisches Krankenhaus, Bethesda Mönchengladbach GmbH, Mönchengladbach, \\ 'Brustzentrum, Ludwig-Maximilians-Universität München, \\ 'Klinik für Gynäkologie und Geburtshilfe, Universitätsklinikum Schleswig-Holstein / Campus Lübeck, Germany
}

\section{Keywords}

Biomarker - Breast cancer - Gene expression .

Prognostic markers · Luminal breast cancer

\section{Summary}

The developments in gene expression analysis have made it possible to sub-classify hormone receptor-positive (luminal) breast cancer in different prognostic subgroups. This sub-classification is currently used in clinical routine as prognostic signature (e.g. 21-gene Onoctype $D X^{\circledR}, 70$-gene Mammaprint $\left.{ }^{\circledR}\right)$. As yet, the optimal method for sub-classification has not been defined. Moreover, there is no evidence from prospective trials. This review explores widely used genomic signatures in luminal breast cancer, making a critical appraisal of evidence from retrospective/prospective trials. It is based on systematic literature search performed using Medline (accessed September 2013) and abstracts presented at the Annual Meeting of American Society of Clinical Oncology and San Antonio Breast Cancer Symposium.

\section{Introduction}

Even with the significant progress in early detection and treatment of hormone receptor (HR)-positive (estrogen receptor (ER)- and/or progesterone receptor (PR)-positive) breast cancer (BC), it remains the most common female cancer in Western Europe, with at least 3 million newly diagnosed cases in 2010, and is still a potentially deadly disease. Currently, the major clinical challenges in $\mathrm{HR}^{+}$luminal $\mathrm{BC}$ are: (1) frequent overtreatment by adjuvant chemotherapy (CT) in about $70 \%$ of cases, based on classical clinical-pathological criteria (tumor size, nodal status, age); (2) selection of patients for extended endocrine therapy (ET) - it was recently shown that ET for 10 years is superior to that for

\author{
Schlüsselwörter \\ Biomarker - Mammakarzinom, luminales . \\ Genexpression - Prognostische Marker
}

\section{Zusammenfassung}

Fortschritte bei Genexpressionsanalysen erlauben eine Einteilung des Hormonrezeptor-positiven (luminalen) Mammakarzinoms in verschiedene prognostische Untergruppen. Diese Subklassifikation wird in der klinischen Routine als prognostische Gensignatur (z.B. 21 Gene/Oncotype $D X^{\circledR}, 70$ Gene/Mammaprint ${ }^{\circledR}$ ) genutzt. Die optimale Methode zur Sub-Klassifizierung ist jedoch noch nicht definiert. Bisher fehlt die Evidenz prospektiver Studien. Diese Übersichtsarbeit analysiert die am weitesten verbreiteten genomischen Signaturen im Sinne einer kritischen Bestandsaufnahme der aus retrospektiven/prospektiven Studien verfügbaren Evidenz. Diese Analyse basiert auf einer systematischen Literaturrecherche mittels Medline, sowie der bis September 2013 veröffentlichten Abstracts beim Annual Meeting of American Society of Clinical Oncology und San Antonio Breast Cancer Symposium.

5 years [1]; and (3) finding predictive factors for targeted therapies that aim to overcome endocrine resistance [2].

The progress in high-throughput analysis of gene expression has led to a substantial change in our understanding of BC. Although HR status was already known as a predictive factor for ET efficacy and as a prognostic factor for BC, the work of Perou et al. [3], and Sorlie et al. [4] demonstrated that $\mathrm{BC}$ is a heterogeneous disease with distinct subtypes, but (sometimes) similar histomorphological characteristics. For the cDNA analysis ( $\mathrm{n}=8,102$ genes), Perou and Sorlie used a small number ( $n=40$, and $n=78$ in the second analysis) of fresh frozen BC samples. Hierarchical clustering analysis revealed 5 molecular subtypes that were relatively stable and intrinsic (mostly driven by genes of the same tumor).

\section{KARGER}

Fax +497614520714

Information@Karger.com

www.karger.com (c) 2013 S. Karger GmbH, Freiburg

$1661-3791 / 13 / 0086-0414 \$ 38.00 / 0$

Accessible online at:

www.karger.com/brc
Dr. Oleg Gluz

Evangelisches Krankenhaus

Bethesda Mönchengladbach $\mathrm{GmbH}$

Ludwig-Weber-Straße 15, 41061 Mönchengladbach, Germany

oleg.gluz@wsg-online.com 
In addition, they were able to correlate these 5 molecular subtypes with differential patient outcome.

This work pointed to ER as a major factor in disease development and its subsequent biological and clinical characteristics. Most $\mathrm{ER}^{+}$cases can be assigned to the luminal A or B subgroup (named after the similarity of their gene expression with that of luminal cells of the normal breast gland). Proliferation-related genes seem to play a major role in the differentiation between both luminal subtypes. Low-proliferating luminal A tumors are associated with better prognosis and lower chemosensitivity than the high-proliferative, frequently poor grade luminal B subtype [4].

The molecular classification according to the definition of Perou et al. is often criticized because of the retrospective nature of the analysis, the small number of tumor samples used for the first analysis $(n=78)$, and the limited number of histological subtypes within small studies. Moreover, although the use of hierarchical clustering as a statistical method is widely applied in hypothesis-generating studies, it is not practicable for clinical use or prospective allocation of patients to the subtypes [5].

\section{Molecular Subtypes}

Allocation of tumors to either luminal subtype seems to be rather less dependable than that for basal-like and HER2 subtypes. Thus, if a nearest centroid predictor is used for allocation, many cases remain unclassified due to the low correlation with the subtype centroid [6]. Weigelt et al. [7] published a substantial lack of reproducibility of luminal subtypes using a single sample predictor method (similarity between subtype centroid and given case). There is only a moderate to weak correlation regarding luminal A or B subtypes between different datasets despite of a similar prognostic impact, thus underlining the mere research character of the molecular classification.

For clinical use, the PAM50 signature (as part of the Prosigna $^{\circledR}$ test, NanoString Inc., Seattle, WA, USA) was developed as an mRNA quantitative reverse transcription PCR based on a 50-gene (and 5 reference genes) assay for sub-classification into the 4 molecular subtypes (luminal A/B, basallike, and HER2). PAM50 is incorporated into a continuous Risk of Relapse (ROR) score [8], which includes the weighting of proliferation genes within molecular subtypes with/ without tumor size (ROR-S/-C). It allocates patients into 1 of 3 risk categories (low $(<10 \%$ distant relapses)/intermediate/ high risk (>20\%) in N0 BC. This signature significantly increases the prognostic impact of the intrinsic subtypes alone [8], and has been shown to be prognostic while showing that the outcome of the luminal B subtype may be quite comparable to basal-like and HER2 subtypes. The prognostic impact of ROR/PAM50 was confirmed in several retrospective analyses of prospective studies. In patients treated by tamoxifen

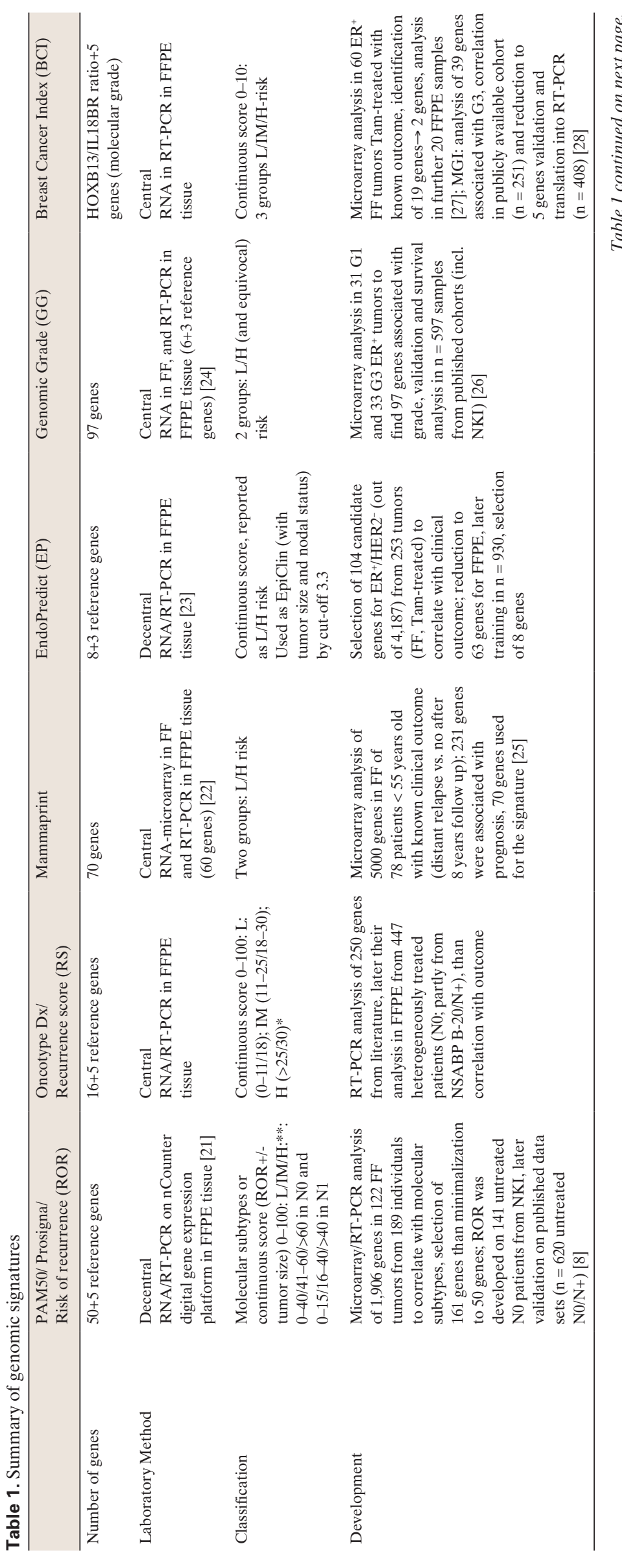




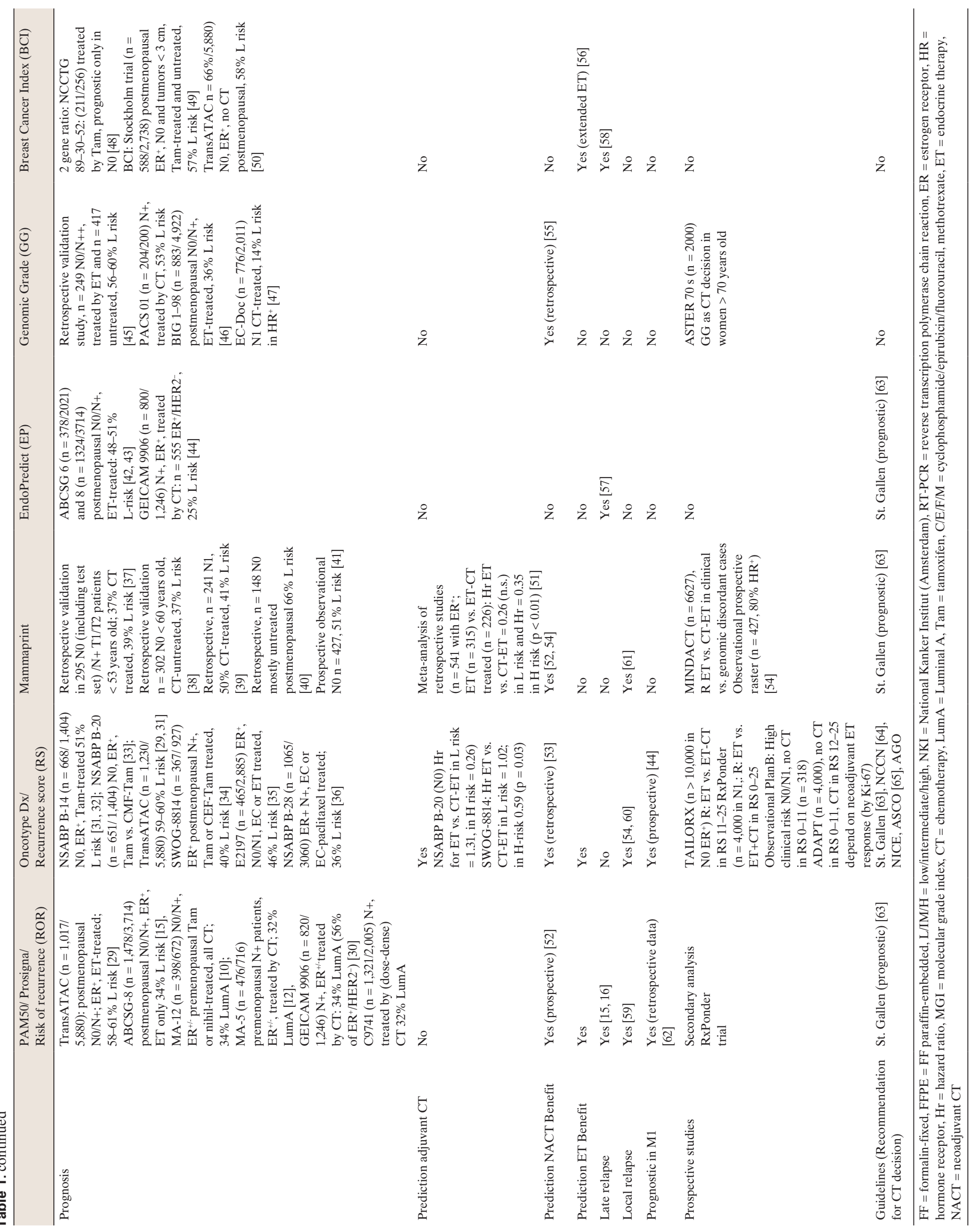


alone, the intrinsic subtype and ROR score were significant prognostic factors together with tumor stage [9]. Moreover, the luminal subtype determined by PAM50 was more predictive for tamoxifen benefit compared to ER status determined by immunohistochemistry (IHC) in premenopausal patients treated within the NCIC MA-12 study [10]. Later, Ellis et al. [11] showed similar activity for neoadjuvant ET in both luminal A/B subtypes. There is some published work on the predictive impact of PAM50 for anthracycline (in the HER2 subgroup) [12] and paclitaxel benefit - surprisingly in the low proliferation group [13] - next to a significant prognostic impact in these smaller retrospective analysis. However, despite strong evidence for a predictive impact of pathological complete response (pCR) within the neoadjuvant CT (NACT) setting, with a 2.3-5.3-fold increase in the pCR rate in the luminal B compared to the luminal A subtype [14], there is still no predictive data for the efficacy of CT + ET versus ET alone. The significant prognostic impact of ROR has recently been retrospectively validated within the TransATAC and ABCSG-08 postmenopausal collectives treated by ET alone. In both studies, ROR provided additional prognostic information beyond clinical variables for both early and late metastasis. Both luminal A and B subtypes and ROR score have shown a prognostic impact over both time periods $(0-5$ and 5-10 years) and provided information in addition to the clinical factors $[15,16]$. A recently presented meta-analysis of the trials in $\mathrm{N}+$ patients confirmed that a large proportion of $\mathrm{N}+$ patients had a low risk (particularly patients with 1-2 positive lymph nodes) [17]. However, the lack of predictive data for the efficacy of adjuvant CT and extended ET, and of decision impact or health economic evaluation studies, are a major consideration for not recommending the PAM50 assay for routine clinical use at the moment. Currently, there are already 2 prospective observational studies of clinical outcomes for the NanoString ${ }^{\circledR}$ Technologies' Breast Cancer Intrinsic Subtype Test (BCIST), 1 in Spain run by GEICAM and 1 in Germany run by WSG (Women's Healthcare Study Group, www.wsg-online.com). Similar studies are planned with separate but also common analyses to look at the health economic impact in different reimbursement systems.

\section{Whole Genome Sequencing}

Besides the controversial discussion regarding standardization of the molecular classification, over the last few years the results of whole genome sequencing of tumors have been published [18]. The luminal subtype (in contrast to basallike tumors) was shown to be characterized by an activated ER-FOXA1 complex. The luminal A subtype (defined by mRNA) harbored significantly more PIK3CA mutations than the luminal B subtype ( $45 \%$ vs. $29 \%$ ), followed by more MAP3K1, and GATA3 and fewer TP53 mutations (12\% vs. $29 \%$ ). However, markers of PIK3CA activation were not elevated in luminal tumors (compared to basal tumors) [19]. GATA3 was also reported to be associated with the aromatase inhibitor (AI) response, and the MAP kinase gene MAP3K1 with both low proliferation levels pre- and post-AI treatment [11]. In the same paper, a significant difference in the number of point mutations was seen between AI-sensitive and -resistant cases.

In addition to PAM50, which aims to standardize the molecular classification of $\mathrm{BC}$, several prognostic gene signatures have been developed for exact prognosis estimation, particularly in $\mathrm{HR}^{+} \mathrm{BC}$ (detailed in table 1). Most of the genes included are involved in proliferation [20], so that to understand their additional prognostic and/or predictive impact compared to classical clinical-pathological or IHC characteristics alone, a comparison with a central and independent pathology assessment of these factors is required.

\section{IHC-Based Classification}

Based on the assumption that proliferation is vital for the differentiation between luminal A and B subtypes, several attempts have been undertaken to replace gene signatures by surrogate IHC markers (e.g. ER, PR, Ki-67, HER2) and/or histological grade assessment [21]. Cheang et al. [87] published a Ki-67 cut-off of $\sim 14 \%$ as an optimal discriminator between luminal A and B subtypes (sensitivity $72 \%$; specificity $78 \%$ ). Therefore, high Ki-67 and/or positive HER2 status were proposed for the definition of luminal B tumors. The biological rationale behind this definition and a correlation with the intrinsic subtype led to the inclusion of the $14 \%$ Ki-67 cut-off into the St. Gallen guidelines in 2011, and to the proposed relative indication for a sequential chemoendocrine therapy in 'luminal B' tumors. However, there is still no consensus about the optimal measurement method and optimal cut-off of Ki-67 [22]. A significant inter-observer variability was confirmed in an international multicenter study (about $1 / 3$ of patients were discordantly allocated to the luminal A vs. B subtypes) [23]. There are several retrospective analyses from prospective trials that confirm a strong prognostic impact of an IHC-based definition of the luminal A and B subtypes [24]. However, 2 recent studies have shown that Ki-67 as a continuous variable (as proposed by the International Consensus) and/or centrally measured grade (as a surrogate for molecular subtype) can provide comparable prognostic information to that derived from any genomic signature $[25,26]$.

Although several papers confirm a predictive impact of high Ki-67 with respect to an increase of pCR in the NACT setting [24], to a benefit from taxanes in addition to anthracyclines in the adjuvant setting $[25,27,28]$ as well as to a benefit of NACT versus ET in the 'luminal B' subtype [29], so far no predictive effect of Ki-67 for benefit of adjuvant CT+ET versus ET alone has been shown [30]. This is difficult to understand in view of the prognostic impact of pCR particularly in 
the luminal B-like but not in the luminal A disease [31]. Because of these methodological difficulties, the most recent St. Gallen Consensus Panel in 2013 voted for changing the Ki-67 cut-off to $20 \%$ (used by most groups for analysis) as an 'IHC-surrogate' definition of the luminal B subtype [32].

IHC scores such as IHC4, which uses centrally determined ER, PR, HER2, Ki-67, or Mammostrat [33], are highly effective methods for classifying $\mathrm{HR}^{+} \mathrm{BC}$ into prognostic subgroups. Dowsett et al. [34] published a similar prognostic impact of the IHC4 score compared to Oncotype DX ${ }^{\circledR}$ (Genomic Health Inc., Redwood City, CA, USA), and the ROR score. However, this could not be confirmed by other groups for the comparison of ROR versus IHC4 or IHC-based subtype [35]. Interestingly, Prat et al. [35] proposed the inclusion of PR $>20 \%$ into the luminal A definition. Other data from the WSG EC-Doc trial also reported a highly significant impact of PR expression within the luminal B subtype for the definition of 2 groups with distinct outcome (i.e. luminal B tumors with high PR expression have an outcome similar to the luminal A subtype) [36].

In summary, despite of methodical criticism, IHC-determined Ki-67, ER, and PR can be used in clinical routine primarily as a prognostic tool (but not as predictive tool for adjuvant CT), particularly if there is access to central pathology experienced in this field. Moreover, they seem to have an important impact as dynamic markers (e.g. drop of Ki-67 and/ or loss of PR) and indicators for efficacy of ET, as they are currently used in the WSG ADAPT [37] and POETIC trials.

\section{Prognostic and Predictive Multigene Signatures}

The 21-gene Oncotype DX assay was developed on the basis of retrospective analyses across 3 studies of 447 patients (all $\mathrm{N}+$ and $\mathrm{N} 0$ ). This analysis was followed by a validation study in tamoxifen-treated N0 $\mathrm{ER}^{+}$patients from the NSABP B-14 study [38]. Distant relapse (DR) was observed in $6.8 \%$ in the low- (L-) and in $30.5 \%$ of the high- (H-) risk groups by recurrence score (RS). A second validation study in N0 patients was conducted within the NSABP B-20 collective. There was no difference in DR after 10 years in the L- (-/+ CT: 96.8/95.6\%) and intermediate- (IM-) risk groups (-/+ CT: $90.9 / 89.1 \%)$. The H-risk group derived a large benefit from adjuvant CT (10 year distant disease-free survival (distant DFS): 60.5 vs. $88.1 \%$; hazard ratio $=0.26$ ) [39]. 2 analyses compared the predictive value of RS and Adjuvant!Online with only high RS being a predictive marker for the benefit of adjuvant CT in N0 disease $[39,40]$ - a finding that was also previously shown in the context of NACT [41]. The prognostic impact of RS was also confirmed in N+ET-treated populations in the TransATAC and SWOG-8814 trials (L vs. H risk: $17-40 \%$ vs. $49-57 \%$ DR after 9-10 years). The differences in the $\mathrm{N}+$ subgroup were mostly driven by the cohort with 4 positive lymph nodes, who formed a rather high percentage of the population in the SWOG trial (38\% vs. $21 \%)$ [42, 43]. The 10 -year overall survival (OS) rates were equal in both studies (L vs. $\mathrm{H}$ risk: $77 \% / 74 \%$ vs. $51 \% / 54 \%$ ). Nevertheless, the most important finding from this analysis was the predictive effect of RS for the benefit of CT-ET compared to ET alone in the RS H-risk group (hazard ratio $=0.59, \mathrm{p}=0.033$ ), but not in the RS L-risk group (hazard ratio $=1.02, p=0.97$ ). None of the conventional prognostic factors were predictive for CT benefit by interaction analysis if RS was included. Curves for the benefit from CT (in the N1 and N2 subgroup) started to split at an RS of 20 (for the 0-5-year endpoint). In this study and in subsequent analyses, the prognostic and predictive impact of RS was stronger in the first 5 years of follow up than in years 5-10. There is further retrospective evidence from prospective trials, such as the E2197 [44] and NSABP B-28 studies, confirming a strong significant prognostic impact in addition to conventional markers, although no predictive impact with respect to paclitaxel CT was shown in the later trial [45].

Furthermore, several studies have indicated a prognostic impact of 21-gene RS, as shown by a good correlation between RS and loco-regional relapse (LRR). There was a very low LRR rate in the L- compared to the H-risk group, with: $1-4 \%$ versus $10-18 \%$, respectively [46, 47]. A strong prognostic impact in primary metastatic $\mathrm{ER}^{+} \mathrm{BC}-$ as the last step of $\mathrm{BC}$ development - has recently been presented, showing a 2-year OS of $100 \%$ in the L-risk $\mathrm{HR}^{+} / \mathrm{HER} 2$ group compared with $69 \%$ in the H-risk group $(n=70)$ [48].

Combination of Oncotype DX with further clinical factors (tumor size, age, grade), i.e. RS-pathological-clinical (RSPC), can increase the prognostic value of the test and reduce number of patients classified as L risk (with/without RSPC: $17.8 \%$ vs, $26.7 \%$ ). However, it was less predictive by interaction analysis with regards to a CT benefit in the NSABP B-20 trial [40].

There are several decision impact studies from different countries evaluating the impact of RS in clinical routine. A change in CT decisions was reported in about $1 / 3$ of patients in the pooled analysis, confirming L risk in $49 \%$ of cases $(\mathrm{n}=1,437)$ [49]. RS has been evaluated in several randomized trials with first prospective evidence expected from the TailorX trial in 2015. This trial (2007-2011) aimed to confirm a 94\% 5-year DFS in the L-risk group and investigate the CT impact in the IM risk group (RS 12-25) in N0 disease. The ongoing RxPonder trial is addressing the question of CT + ET versus ET alone in N1 L and IM risk by RS (RS 0-25) patients with N1 BC $(n=4,000)$. The WSG ADAPT trial is investigating the CT decision in the N0-1 IM risk by RS depending on early ET response ( $\mathrm{n}=\sim 1,600$ by ET alone). This is a followup trial to the planB trial (2009-2011) in which 318 patients with clinical IM or $\mathrm{H}$ risk and low RS opted for omission of CT.

The NCCN [50], ASCO [51], and current St. Gallen Consensus Panel [32] have recommended that the use of RS be 
considered in the decision whether $\mathrm{CT}$ is given in $\geq \mathrm{pT} 1 \mathrm{~b}$ N0 and N1mi disease.

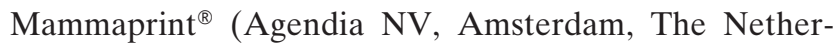
lands) is a 70-gene prognostic signature that was developed in 2002, and has been recently been complemented by the BluePrint $^{\circledR}$ (Agendia NV, for molecular subtype) and TargetPrint ${ }^{\circledR}$ (Agendia NV) assays (for ER, PR, HER2). Based on the expression of 70 genes, patients are allocated to the $\mathrm{L}$ - or H-risk subgroups, respectively (in both $\mathrm{HR}^{+}$and $\mathrm{HR}^{-} \mathrm{BC}$ ). A subsequent validation study (including patients from the original study) provided a clear correlation between risk assessment and relapse risk in 153 N0, mostly CT-naïve, patients (L vs. H risk: $13 \%$ vs. $56 \%$ after 5 years) [52]. A second validation study in N0 BC showed a 10-year OS in L- versus H-risk subgroups of $88 \%$ and $71 \%$, respectively [53]. About $1 / 3$ of patients were discordant by clinical and genomic assessment, so that CT could only be spared effectively in $20 \%$. Later studies have shown a strong prognostic impact in N1 BC ( $\mathrm{n}=241$, $\sim 50 \%$ treated by CT; 5 -year DFS $98 \%$ vs. $80 \%$ in L- vs. $\mathrm{H}$ - risk subgroups) [54], and also in postmenopausal N0 patients [55]. The 10-year LRR was reported as $5.7 \%$ versus $13.5 \%$ in the L- compared with the H-risk subgroups, respectively $(\mathrm{n}=1,053)$ [56]. Knauer et al. [57] performed a metaanalysis of the predictive impact for adjuvant CT for 7 retrospective studies. The analysis showed a hazard ratio of 0.26 for 5-year DFS in the L-risk subgroup (ET vs. ET + CT: 93\% vs. $99 \%, p=0.2)$ and a hazard ratio of 0.35 (5-year DFS 76\% vs. $88 \%, p<0.01)$ in the H-risk subgroup. Meta-analysis data of prospective and retrospective studies [58] from the NACT setting showed a higher pCR rate (11\% vs. $6 \%)$ after NACT in $\mathrm{H}$ - versus L-risk $\mathrm{HR}^{+}$patients, and a prognostic impact of pCR in H-risk disease but a better survival in the L-risk BC [59]. The prospective observational RASTER study revealed a better 5-year distant-recurrence-free survival (DRFS) in the L- compared to the H-risk subgroup (97\% vs. 91.7\%), which was independent of Adjuvant!Online allocation after 61 months of median follow-up [60].

The prospective randomized MINDACT trial, which directly compared genomic and clinical assessments, has been completed $(\mathrm{n}=6,527)$. Discordance in risk assessment was reported in $32 \%$ of all cases, but CT could only be spared in $11.2 \%$ of all cases (per protocol) [61].

The EndoPredict ${ }^{\circledR}$ test (Sividon Diagnostics GmbH, Cologne, Germany) is another RNA-based tool for $\mathrm{ER}^{+} \mathrm{BC}$ that is widely used in German-speaking countries. It can be combined with clinical-pathological factors (tumor size and nodal status) into an EPClin score and divides patients into an L- or H-risk category even though the score itself is a continuous variable. The test was developed in mostly L-risk ER ${ }^{+} / \mathrm{HER}^{-}$ patients receiving ET alone ( $65 \%$ N0, 10\% G3). Pre-specified cut-offs were validated on samples from the ABCSG-6 and -8 studies, which included clinically L-risk postmenopausal patients (mostly G1/2, N0) treated by ET alone [62, 63].

At pre-specified cut-offs of EPclin, the 10-year risk for DR is $4 \%$ compared with $22-28 \%$ in the L- versus the H-risk group, respectively. The test identifies only $13-17 \%$ of 'luminal B' tumors as L risk, but provides additional prognostic information to clinical and IHC markers (Ki-67 and ER). It is reported to be prognostic for early as well as late recurrences. However, there is a distinct biology of early and late recurrences with the ER-related gene group (but not the proliferation group), which is associated with relapses in years 5-10 [64].

The test is listed by the St. Gallen Consensus [32] because of its strong prognostic data, but may not be recommended at the moment for the decision on $\mathrm{CT}$ in $\mathrm{HR}^{+} \mathrm{BC}$ due to the absent predictive data for adjuvant $\mathrm{CT}$.

Genomic Grade $^{\circledR}$ (GG, bioTheranostics Inc., San Diego, CA, USA) is primarily a 97-gene signature in $\mathrm{HR}^{+} \mathrm{BC}$, which was published by Sotiriou et al. in 2006 [65]. The aim of the original work was to correlate known gene expression data with histological grade (HG) 1 and 3 tumors. Most of the differentially expressed genes were shown to be related to proliferation. Loi et al. [66] published a validation study, with a 10-year DRFS survival in GG1 of $83 \%$ in ET-treated N0/N+ patients. Later work demonstrated a better prognostic performance of GGI compared with Ki-67 IHC, mRNA, and mitotic count in a small cohort of N+CT-treated patients from the PACS01 study (5-year DFS GG1 vs. GG3: 89\% vs. 64\%) [67]. Liedtke et al. [68] also reported predictive data for a better response to NACT but also worse survival in GG3 versus GG1 in $\mathrm{HR}^{+}$tumors (but not $\mathrm{HR}^{-} \mathrm{BC}$ ).

Two further prospective-retrospective studies were presented last year. Sotiriou et al. [26] compared the prognostic impact of GGI and Ki-67 in the BIG 1-98 monotherapy collective, with $40 \%$ of tumors identified as equivocal by GG analysis (39\% pCR failure rate). In the N0 ET alone cohort, $99 \%$ a 10 -year DFS was seen in GG1 compared to $87 \%$ in GG3 tumors. However, there was a comparable prognostic impact of both $\mathrm{Ki}-67$ and $\mathrm{GG}$ as continuous variables in this cohort. Very similar data from the WSG EC-Doc study have also been reported (39\% RNA failure rate; $76 \%$ GG3). GG was prognostic in multivariate analysis (including central grade), if used as a continuous, but not if used as a dichotomous, variable. IHC luminal B subtype was a better predictive factor for taxane benefit [25].

The Breast Cancer Index ${ }^{\circledR}$ (BCI, bioTheranostics Inc.) consists of the HOXB13/IL18BR gene expression ratio, which is prognostic [69] and predictive for ET benefit [70], and of the molecular grade index (MGI), which is a set of cell cyclerelated genes. The gene expression ratio predicted outcome in untreated and in ET-treated patients better than conventional markers. The MGI, as a reflection of the 97-gene GG, was shown to provide complementary information to the GG [71]. The combination of both markers was validated in the Stockholm trial and a continuous BCI was established [72]. DFS at 10 years was $3 \%$ in the Tam-treated group and $50 \%$ in the untreated group. Interestingly, BCI was powerful in the de- 
tection of both early and late recurrences as the only prognostic factor in the multivariate analysis, most likely due to the fact that it combines both ER- and proliferation-related factors [73]. This finding is of immediate clinical significance since, in a nested case-control study, HOXB13/IL18BR identified patients in the MA-17 trial who benefitted from extended ET by letrozole (83 recurrences vs. 166 non-recurrences) [74]. Identification of late recurrences was also assessed within the TransATAC trial. L-risk patients had a 10year DR of $4.2 \%$ (compared to $17 \%$ of patients in the H-risk group with a $30 \%$ DR). Although the prognostic impacts of $\mathrm{BCI}, \mathrm{RS}$, and ROR were similar over the first 5 years, only $\mathrm{BCI}$ was predictive for late recurrences over the following 5-10 years.

Last but not least, the sensitivity to the ET index (SET) needs to be mentioned here as an example of second generation signatures. Symanns et al. [75] identified 165 genes coregulated with ER. The signature is predictive of ER pathway activity and has a prognostic impact in patients receiving ET (with/without NACT, $\mathrm{n}=523$ ), but not in untreated patients $(\mathrm{n}=341)$.

\section{Concordance Between Prognostic Tools}

The current St. Gallen Consensus recommends the use of multigene signatures particularly in luminal B disease for selection of patients who should undergo adjuvant CT [32]. As mentioned previously, there is only weak to moderate concordance between genomic tools and IHC classification of $\mathrm{HR}^{+}$BC. Prat et al. [35] reported $\mathrm{k}$ values of $0.2-0.41$ for IHC versus PAM50 (in the earlier work $\mathrm{k}=0.47$ [9]), and 35-52\% of IHC luminal B tumors were re-classified to luminal A using PAM50. It is important to state that the PAM50/ROR score provided additional prognostic information to that of the IHC subtype and IHC4, but the converse was not true. Similar data have been reported for the MINDACT trial, where only a $71 \%$ concordance between luminal subtypes by BluePrint and IHC (Ki-67 cut-off of 14\%) was detected. In this study, 61\% of 'IHC-based luminal B subtypes' were classified as L-risk luminal A by BluePrint [76].

The prospective planB study showed a comparable correlation between central grade, Ki-67 and RS ( $\mathrm{n}=2,566) .11 \%$ of luminal B (Ki-67 $\geq 20 \%$ ) tumors were L risk and 48\% IM risk by RS. The correlation between central grade and RS was poorer ( $\mathrm{k}$ of 0.32 ) [77]. Only a moderate correlation of $\mathrm{R}_{\mathrm{s}}=0.65$ was found between Oncotype DX and EndoPredict (Sividon Diagnostics GmbH, Cologne, Germany) in a small study, due mainly to $61 \%$ of H-risk patients by EndoPredict being classified as L or IM risk by RS [78].

Depending on different patient selection criteria, good concordance between H-risk assessments by the different genomic signatures seems to exist, but there are slightly divergent results in the L- and IM-risk groups. The prognostic value of a multigene assay is strongly dependent on the number of patients allocated into the IM-risk group and whether further clinical markers are included. Recently, Dowsett et al. [34] reported high concordance between L- and H-risk assessments ( $73 \%$ and $67 \%$, respectively) obtained by ROR and RS. However, there was only a weak concordance in the IMrisk group (35\%). Again, due to slightly different numbers in the IM-risk group, there was high prognostic concordance in the H- and L-risk groups, but less so in the IM-risk subgroups. ROR added some prognostic information to the RS, but the converse was not true.

Prat et al. [79] reported a consistent prognostic impact of all signatures (ROR, RS, Mammaprint, SET) as independent markers in the multivariate analysis (despite of a very low overlap between the individual genes, $<25 \%$ ), so that a combination of these signatures increased the performance. ROR and RS showed the highest concordance. The results of the RxPonder trial are needed before final conclusions regarding a direct comparison between ROR-Score and RS can be drawn. This is a secondary objective of the trial.

\section{Conclusions}

The various genomic signatures presented in this review reflect the heterogeneity of $\mathrm{HR}^{+}$early $\mathrm{BC}$ and its distinct prognostic groups in a highly reproducible way. Valid analytical data are available for most of these signatures (e.g. for Oncotype DX, Mammaprint, EndoPredict, PAM50), and show: a high correlation between the same patients samples [80]; a high inter-laboratory correlation [53, 81, 82]; and good correlations between core biopsies and whole block samples $[83,84]$. Although a multivariate prognostic effect of such genomic tools has been shown in most retrospective studies, it should be noted that several analyses did not include important prognostic variables such as Ki-67 and/or PR. Despite of their controversial reproducibility, inclusion of independently and centrally determined IHC factors is definitely required before a general recommendation to widely use genomic markers can be made. The combination with clinical markers seems to increase the prognostic value of these multigene tests $[8,40,62]$, but may lead to a relative loss of their predictive impact.

To summarize the decision impact of genomic signatures, it is also important to remember that, despite a decision to change treatment in $30-40 \%$ of cases, absolute sparing of CT only occurred in about $10-13 \%$ of patients in most retrospective [85] and prospective $[61,86]$ studies. Combination of a genomic signature (Oncotype DX) with response to shortterm preoperative ET within the WSG ADAPT trial [37] may increase this CT-sparing percentage up to $35-40 \%$ in patients with an originally strong indication for CT.

The IMPAKT 2012 Working Group Consensus Statement found the analytical and clinical validity of Oncotype DX and 
Mammaprint convincing. However, none of the tests has shown robust clinical utility so far. Results of prospective trials (TAILORX, RxPOnder, MINDACT, planB, ADAPT) are eagerly awaited for clarification of the clinical impact of genomic tools within $\mathrm{HR}^{+} \mathrm{BC}$. Oncotype $\mathrm{DX}$ is recommended by the 2013 St. Gallen Consensus for making an adjuvant CT decision based on its predictive data. In Germany, the German Arbeitsgemeinschaft Gynäkologische Onkologie (AGO) still recommends the use of molecular tools only in individual cases and within clinical trials. Nevertheless, the signatures discussed in this manuscript all have the potential to support clinical decision making in early BC, and thus to help both avoiding overtreatment by adjuvant $\mathrm{CT}$ as well as undertreatment.

\section{Disclosure Statement}

Oleg Gluz received honoraria (advisory board) from Genomic Health Inc. and Hanostring Inc. Daniel Hofmann has no conflicts of interest to disclose.

\section{References}

1 Davies C, et al.: Long-term effects of continuing adjuvant tamoxifen to 10 years versus stopping at 5 years after diagnosis of oestrogen receptor-positive breast cancer: ATLAS, a randomised trial. Lancet 2013;381:805-816.

2 Baselga J, et al.: Everolimus in postmenopausal hormone-receptor-positive advanced breast cancer. N Engl J Med 2012;366:520-529.

$\checkmark 3$ Perou CM, et al.: Molecular portraits of human breast tumours. Nature 2000;406:747.

4 Sorlie T, et al.: Gene expression patterns of breast carcinomas distinguish tumor subclasses with clinical implications. Proc Natl Acad Sci USA 2001; 98:10869-10874.

5 Mackay A, et al.: Microarray-based class discovery for molecular classification of breast cancer: analysis of interobserver agreement. J Natl Cancer Inst 2011;103:662-673.

6 Sorlie T, et al.: Repeated observation of breast tumor subtypes in independent gene expression data sets. Proc Natl Acad Sci USA 2003;100:84188423.

7 Weigelt B, et al.: Breast cancer molecular profiling with single sample predictors: A retrospective analysis. Lancet Oncol 2010;11:339.

8 Parker JS, et al.: Supervised risk predictor of breast cancer based on intrinsic subtypes. J Clin Onco 2009;27:1160-1167.

$\checkmark 9$ Nielsen TO, et al.: A Comparison of PAM50 intrinsic subtyping with immunohistochemistry and clinical prognostic factors in tamoxifen-treated estrogen receptor-positive breast cancer. Clin Cancer Res 2010;16:5222-5232.

10 Chia SK, et al.: A 50-Gene intrinsic subtype classifier for prognosis and prediction of benefit from adjuvant tamoxifen. Clin Cancer Res 2012;18:44654472.

11 Ellis M, et al.: ACOSOG Z1031: A randomized neoadjuvant comparison between letrozole (LET), anastrozole (ANA) and exemestane (EXE) for postmenopausal women with ER rich stage $2 / 3$ breast cancer: Biomarker outcomes and the predictive value of the baseline PAM50 based intrinsic subtype. Cancer Res 2011;71(suppl 1):S1-2.

12 Cheang MCU, et al.: Responsiveness of intrinsic subtypes to adjuvant anthracycline substitution in the NCIC.CTG MA.5 randomized trial. Clin Cancer Res 2012;18:2402-2412.

13 Martín M, et al.: PAM50 proliferation score as a predictor of weekly paclitaxel benefit in breast cancer. Breast Cancer Res Treat 2013;138:457-466.

14 Esserman L, et al.: Chemotherapy response and recurrence-free survival in neoadjuvant breast cancer depends on biomarker profiles: results from the I-SPY 1 TRIAL (CALGB 150007/150012; ACRIN
6657). Breast Cancer Res Treat 2012;132:10491062.

15 Gnant M, et al.: Prpredicting risk for late metastasis: The PAM50 risk of recurrence (ROR) score after 5 years of endocrine therapy in postmenopausal women with HR+ early breast cancer: A study on 1,478 patients from the ABCSG- 8 trial. Ann Oncol 2013;24(suppl 3):iii29.

16 Sestak I, et al.: Factors predicting late recurrence for estrogen receptor-positive breast cancer. J Natl Cancer Inst 2013;105:1504-1511.

17 Gnant M, et al.: Identifying clinically relevant prognostic subgroups in node-positive postmenopausal HR+ early breast cancer patients treated with endocrine therapy: A combined analysis of 2,485 patients from ABCSG-8 and ATAC using the PAM50 risk of recurrence (ROR) score and intrinsic subtype. ASCO Meeting Abstracts 2013; 31(15_suppl):506.

18 Ding L, et al.: Genome remodelling in a basal-like breast cancer metastasis and xenograft. Nature 2010;464:999-1005.

19 Cancer Genome Atlas Network. Comprehensive molecular portraits of human breast tumours. Nature 2012;490:61-70.

20 Wirapati P, et al.: Meta-analysis of gene expression profiles in breast cancer: Toward a unified understanding of breast cancer subtyping and prognosis signatures. Breast Cancer Res 2008;10:R65.

21 Goldhirsch A, et al.: Strategies for subtypes - dealing with the diversity of breast cancer: highlights of the St Gallen International Expert Consensus on the Primary Therapy of Early Breast Cancer 2011. Ann Oncol 2011;22:1736-1747.

22 Dowsett M, et al.: Assessment of Ki67 in Breast Cancer: Recommendations from the International Ki67 in Breast Cancer Working Group. J Natl Cancer Inst 2011;103:1656-1664.

23 Nielsen T, et al.: An international Ki67 reproducibility study. Cancer Res 2012;72(24 suppl):S4-6.

24 Denkert C, et al.: Ki67 levels as predictive and prognostic parameters in pretherapeutic breast cancer core biopsies: A translational investigation in the neoadjuvant GeparTrio trial. Ann Oncol 2013;24:2786-2793.

25 Gluz O, et al.: The prognostic and predictive impact of genomic grade index (GGI) versus central grade or molecular class in intermediate-risk breast cancer (BC): Results from the EC-Doc trial. ASCO Meeting Abstracts 2013;31(15_suppl):1092.

26 Sotiriou C, et al.: Independent validation of genomic grade in the BIG 1-98 study. Cancer Res 2012;72(24 suppl):S4-4.

27 Hugh J, et al.: Breast cancer subtypes and response to docetaxel in node-positive breast cancer: Use of an immunohistochemical definition in the BCIRG 001 trial. J Clin Oncol 2009;27:1168-1176.

28 Penault-Llorca F, et al.: Ki67 Expression and docetaxel efficacy in patients with estrogen receptorpositive breast cancer. J Clin Oncol 2009;27:28092815.

29 Alba E, et al.: Chemotherapy (CT) and hormonotherapy (HT) as neoadjuvant treatment in luminal breast cancer patients: Results from the GEICAM/ 2006-03, a multicenter, randomized, phase-II study. Ann Oncol 2012;23:3069-3074.

30 Viale G, et al.: Prognostic and predictive value of centrally reviewed Ki-67 labeling index in postmenopausal women with endocrine-responsive breast cancer: Results from Breast International Group Trial 1-98 comparing adjuvant tamoxifen with letrozole. J Clin Oncol 2008;26:5569-5575.

31 Von Minckwitz G, et al.: Correlation of various pathologic complete response ( $\mathrm{pCR}$ ) definitions with long-term outcome and the prognostic value of pCR in various breast cancer subtypes: Results from the German neoadjuvant meta-analysis. J Clin Oncol 2011;29(suppl):abstr 1028.

32 Goldhirsch A, et al.: Personalizing the treatment of women with early breast cancer: Highlights of the St Gallen International Expert Consensus on the Primary Therapy of Early Breast Cancer 2013. Ann Oncol 2013;24:2206-2223.

33 Bartlett JMS, et al.: Mammostrat as an immunohistochemical multigene assay for prediction of early relapse risk in the Tamoxifen versus Exemestane Adjuvant Multicenter Trial Pathology Study. J Clin Oncol 2012;30:4477-4484.

34 Dowsett M, et al.: Comparison of PAM50 risk of recurrence score with oncotype DX and IHC4 for predicting risk of distant recurrence after endocrine therapy. J Clin Oncol 2013;31:2783-2790.

35 Prat A, et al.: Prognostic significance of progesterone receptor-positive tumor cells within immunohistochemically defined luminal A breast cancer. J Clin Oncol 2013;31:203-209.

36 Gluz O, et al.: PR expression in luminal breast cancer. Senology 2013; accepted.

37 Hofmann D, et al.: WSG ADAPT - adjuvant dynamic marker-adjusted personalized therapy trial optimizing risk assessment and therapy response prediction in early breast cancer: study protocol for a prospective, multi-center, controlled, non-blinded, randomized, investigator initiated phase II/III trial. Trials 2013;14:261.

38 Paik S, et al.: A multigene assay to predict recurrence of tamoxifen-treated, node-negative breast cancer. N Engl J Med 2004;351:2817-2826.

39 Paik S, et al.: Gene expression and benefit of chemotherapy in women with node-negative, estro- 
gen receptor-positive breast cancer. J Clin Oncol 2006;24:3726-3734.

40 Tang G, et al.: Risk of recurrence and chemotherapy benefit for patients with node-negative, estrogen receptor-positive breast cancer: recurrence score alone and integrated with pathologic and clinical factors. J Clin Oncol 2011;29:4365-4372.

41 Gianni L, et al.: Gene expression profiles in paraffin-embedded core biopsy tissue predict response to chemotherapy in women with locally advanced breast cancer. J Clin Oncol 2005;23:7265-7277.

42 Dowsett M, et al.: Prediction of risk of distant recurrence using the 21-gene recurrence score in node-negative and node-positive postmenopausal patients with breast cancer treated with anastrozole or tamoxifen: A TransATAC study. J Clin Oncol 2010;28:1829-1834.

43 Albain KS, et al.: Prognostic and predictive value of the 21-gene recurrence score assay in postmenopausal women with node-positive, oestrogen-receptor-positive breast cancer on chemotherapy: a retrospective analysis of a randomised trial. Lancet Oncol 2010;11:55-65.

44 Goldstein LJ, et al.: Prognostic utility of the 21gene assay in hormone receptor-positive operable breast cancer compared with classical clinicopathologic features. J Clin Oncol 2008;26:4063-4071.

45 Mamounas E, et al.: Association between the 21gene recurrence score (RS) and benefit from adjuvant paclitaxel $(\mathrm{Pac})$ in node-positive $(\mathrm{N}+)$, ERpositive breast cancer patients (pts): Results from NSABP B-28. Cancer Res 2012;72(24 suppl):S1-10.

46 Mamounas EP, et al.: Prognostic impact of the 21gene recurrence score (RS) on loco-regional recurrence (LRR) of node-positive, ER-positive breast cancer patients (pts) treated with adjuvant chemotherapy: Results from NSABP B-28. Cancer Res 2012;72(24 suppl):P6-07-01.

47 Solin L, et al.: Prognostic value of biologic subtype and the 21-gene recurrence score relative to local recurrence after breast conservation treatment with radiation for early stage breast carcinoma results from the Eastern Cooperative Oncology Group E2197 study. Breast Cancer Res Treat 2012; 134:683-692.

48 King TA, et al.: Prognostic impact of the 21-gene recurrence score in patients presenting with stage IV breast cancer. ASCO Meeting Abstracts 2013;31(15_suppl):507.

49 Carlson J, et al.: The impact of the oncotype Dx breast cancer assay in clinical practice: A systematic review and meta-analysis. Breast Cancer Res Treat 2013;141:13-22.

-50 Theriault RL, et al.: Breast Cancer, Version 3.2013. J Natl Compr Canc Netw 2013;11:753-761.

-51 Harris L, et al.: American Society of Clinical Oncology 2007 Update of Recommendations for the Use of Tumor Markers in Breast Cancer. J Clin Oncol 2007;25:5287-5312.

52 van 't Veer LJ, et al.: Gene expression profiling predicts clinical outcome of breast cancer. Nature 2002;415:530

53 Buyse M, et al.: Validation and clinical utility of a 70 -gene prognostic signature for women with nodenegative breast cancer. J Natl Cancer Inst 2006; 98:1183-1192.

54 Mook S, et al.: The 70-gene prognosis-signature predicts disease outcome in breast cancer patients with 1-3 positive lymph nodes in an independent validation study. Breast Cancer Res Treat 2009; 116:295.
55 Mook S, et al.: The 70-gene prognosis signature predicts early metastasis in breast cancer patients between 55 and 70 years of age. Ann Oncol 2010;21:717-722.

56 Drukker C, et al.: Gene expression profiling to predict the risk of locoregional recurrence in breast cancer. Cancer Res 2012;72(24 suppl):P2-10-42.

57 Knauer M, et al.: The predictive value of the 70gene signature for adjuvant chemotherapy in early breast cancer. Breast Cancer Res Treat 2010; 120:655-661.

58 Esserman LJ, et al.: Pathologic complete response predicts recurrence-free survival more effectively by cancer subset: Results from the I-SPY 1 TRIAL_CALGB 150007/150012, ACRIN 6657. J Clin Oncol 2012;30:3242-3249.

59 Gluck S, et al.: Response and long-term outcomes after neo-adjuvant chemotherapy: Pooled dataset of patients stratified by molecular subtyping using MammaPrint and BluePrint. Cancer Res 2012; 72(24 suppl):P3-06-11.

60 Drukker CA, et al.: A prospective evaluation of a breast cancer prognosis signature in the observational RASTER study. Int J Cancer 2013;133:929936.

61 Piccart M, et al.: The EORTC 10041/BIG 03-04 MINDACT (microarray in node negative and 1 to 3 positive lymph node disease may avoid chemotherapy) Trial: Patients' Baseline Characteristics and Logistics Aspects After a Successful Accrual. Eur J Cancer 2011;47(suppl 2):7.

62 Filipits M, et al.: A new molecular predictor of distant recurrence in ER-positive, HER2-negative breast cancer adds independent information to conventional clinical risk factors. Clin Cancer Res 2011:17:6012-6020.

63 Dubsky P, et al.: EndoPredict improves the prognostic classification derived from common clinical guidelines in ER-positive, HER2-negative early breast cancer. Ann Oncol 2013;24:640-647.

64 Dubsky P, et al.: The EndoPredict score identifies late distant metastases in ER+/HER2- breast cancer patients. Cancer Res 2012;72(24 suppl):S4-3

65 Sotiriou C, et al.: Gene expression profiling in breast cancer: Understanding the molecular basis of histologic grade to improve prognosis. J Natl Cancer Inst 2006;98:262-272.

66 Loi S, et al.: Definition of clinically distinct molecular subtypes in estrogen receptor-positive breast carcinomas through genomic grade. J Clin Oncol 2007;25:1239-1246.

67 Bertucci F, et al.: Comparison of the prognostic value of genomic grade index, Ki67 expression and mitotic activity index in early node-positive breast cancer patients. Ann Oncol 2013;24:625-632.

68 Liedtke C, et al.: The Genomic Grade Index (GGI) is associated with response to neoadjuvant chemotherapy in patients with breast cancer. J Clin Oncol 2009;27:3185-91.

69 Goetz MP, et al.: A two-gene expression ratio of homeobox 13 and interleukin-17B receptor for prediction of recurrence and survival in women receiving adjuvant tamoxifen. Clin Cancer Res 2006;12:2080-2087.

70 Ma X.-J, et al.: The HOXB13:IL17BR expression index is a prognostic factor in early-stage breast cancer. J Clin Oncol 2006;24:4611-4619.

71 Ma X.-J, et al.: A five-gene molecular grade index and HOXB13:IL17BR are complementary prognostic factors in early stage breast cancer. Clin Cancer Res 2008;14:2601-2608.
72 Jerevall PL, et al.: Prognostic utility of HOXB13:IL17BR and molecular grade index in early-stage breast cancer patients from the Stockholm trial. Br J Cancer 2011;104:1762-1769.

73 Zhang Y, et al.: Breast cancer index identifies early-stage estrogen receptor-positive breast cancer patients at risk for early- and late-distant recurrence. Clin Cancer Res 2013;19:4196-4205.

74 Sgroi DC, et al.: Prediction of late disease recurrence and extended adjuvant letrozole benefit by the HOXB13/IL17BR biomarker. J Natl Cancer Inst 2013;105:1036-1042.

75 Symmans WF, et al.: Genomic index of sensitivity to endocrine therapy for breast cancer. J Clin Oncol 2010;28:4111-4119.

76 Viale G, et al.: Pathological assessment of discordant cases for molecular (BluePrint and MammaPrint) versus clinical subtypes for breast cancer among 621 patients from the EORTC 10041/BIG 3-04 (MINDACT) trial. Cancer Res 2012;72(24 suppl):P3-05-02.

77 Gluz O, et al.: S4-3: Prospective comparison of risk assessment tools in early breast cancer (Recurrence Score, uPA/PAI-1, central grade, and luminal subtypes): Final correlation analysis from the phase III WSG-Plan B Trial. Cancer Res 2012; 71(24 suppl):S4-3.

78 Varga Z, et al.: Comparison of EndoPredict and Oncotype DX test results in hormone receptor positive invasive breast cancer. PLoS One 2013; 8:e58483.

79 Prat A, et al.: Concordance among gene expression-based predictors for ER-positive breast cancer treated with adjuvant tamoxifen. Ann Oncol 2012;23:2866-2873.

80 Habel L, et al.: A population-based study of tumor gene expression and risk of breast cancer death among lymph node-negative patients. Breast Cancer Res 2006;8:R25.

81 Ach R, et al.: Robust interlaboratory reproducibility of a gene expression signature measurement consistent with the needs of a new generation of diagnostic tools. BMC Genomics 2007;8:148.

82 Kronenwett R, et al.: Decentral gene expression analysis: analytical validation of the Endopredict genomic multianalyte breast cancer prognosis test. BMC Cancer 2012;12:456.

83 Drury S, et al.: Feasibility of using tissue microarray cores of paraffin-embedded breast cancer tissue for measurement of gene expression: A proofof-concept study. J Clin Pathol 2010;63:513-517.

84 Müller BM, et al.: Comparison of the RNA-based EndoPredict multigene test between core biopsies and corresponding surgical breast cancer sections. J Clin Pathol 2012;65:660-662.

85 Müller BM, et al.: The EndoPredict gene-expression assay in clinical practice - performance and impact on clinical decisions. PLoS One 2013; 8:e68252.

86 Albanell J, et al.: Prospective transGEICAM study of the impact of the 21-gene recurrence score assay and traditional clinicopathological factors on adjuvant clinical decision making in women with estrogen receptor-positive $(\mathrm{ER}+)$ node-negative breast cancer. Ann Oncol 2012;23:625-631.

87 Cheang MC, et al.: Ki67 index, HER2 status, and prognosis of patients with luminal B breast cancer. J Natl Cancer Inst 2009;101:736-750. 